\title{
Conception et analyse de dispositifs d'investigation en biologie: comment conjuguer autonomie dans la validation scientifique, approfondissement conceptuel dans le paradigme et couverture curriculaire?
}

\section{François Lombard}

L'investigation est un modèle pédagogique imposé en France et encouragé en Europe pour l'enseignement des sciences. Il est fondé sur la dévolution des questions aux élèves, mais cette exigence est souvent en tension avec la nécessité de couvrir le curriculum et de garantir que les élèves acquièrent des connaissances scientifiques approfondies. Nous avons conçu un dispositif d'investigation basé sur un espace d'écriture partagé en biologie au secondaire supérieur, développé durant 10 ans. Nous avons analysé quatre années pour en extraire une conceptualisation, une description abstraite et l'identification des variables pertinentes (complexité épistémique). Dans une approche méthodologique Design-Based-Research, nous avons analysé les traces des productions écrites des élèves, des questionnaires en fin d'année et un an après à l'université. Nous proposons notamment la distinction entre l'autorité pédagogique et l'autorité scientifique, ainsi que 27 recommandations de design éprouvées. Leur synthèse révèle l'interdépendance de trois variables: i) la responsabilité assumée par les élèves de produire chacun une part des savoirs, ii) le but commun d'approfondir les connaissances et iii) le rôle de ressources authentiques pour guider l'affinage conceptuel des questions.

L'apprentissage des sciences est confronté à plusieurs difficultés et problématiques. Dans le secondaire, nous relevons notamment la difficulté à conduire les élèves vers des connaissances approfondies. En biologie, relevons notamment la compréhension des mécanismes sous-jacents aux phénomènes étudiés, l'application extrascolaire dans la perspective de rendre les élèves capables de comprendre les enjeux scientifiques, d'utiliser les outils de la science pour comprendre le monde qui les entoure et de prendre en compte et de mettre en perspective les connaissances scientifiques pour construire leurs décisions citoyennes (Rocard et al., 2006). Selon Popper, «le fait est que notre pédagogie consiste à submerger les enfants de réponses à des questions qu'ils ne se sont pas posées alors qu'on n'écoute pas les questions qu'ils posent» (Popper, 1990, p. 63).

Sans faire ici le débat sur les modèles d'apprentissage et les pédagogies correspondantes, nous sommes d'accord avec Kirschner, Sweller et Clark (2006) sur 
la nécessité d'un guidage de l'apprenant, mais poursuivons la réflexion avec la synthèse de Tobias \& Duffy (2009) qui montre qu'entre tenants et opposants des «approches constructivistes», il y a accord sur la nécessité de guider les élèves et étayer l'apprentissage, mais ce sont les formes de ce guidage qui font débat. Dans cet article, nous ne cherchons pas à valider le modèle pédagogique d'investigation, nous prenons acte qu'il fait l'objet de nombreuses recherches, qu'il est imposé en France et encouragé en Europe pour l'enseignement des sciences et que donc de nombreux enseignants tentent de le mettre en œuvre. Nous nous limiterons à explorer et discuter les liens entre la structure d'un dispositif d'investigation et les effets éducatifs afin de proposer des recommandations éprouvées qui pourraient aider à la conception et au guidage de dispositifs d'enseignements diversifiés.

L'objectif de notre thèse était: «Comment aider élèves à développer des connaissances scientifiques approfondies en biologie dans des environnements infodenses?"

L'investigation est fondée sur la dévolution des questions ce qui rend difficile le pilotage de l'investigation à l'intérieur du curriculum et le guidage vers l'approfondissement. C'est cet aspect que nous discuterons spécifiquement dans cet article. Nous allons donc poser ici quelques éléments du cadre théorique, de la méthodologie, présenter une sélection parmi les résultats et une synthèse des conclusions.

Une problématique cruciale dans l'enseignement des sciences concerne la difficulté à mettre en œuvre et distinguer les formes d'autorité(s) puisque, d'un côté, le scepticisme scientifique refuse l'argument d'autorité mais, de l'autre, l'enseignant doit être garant des objectifs curriculaires et doit donc faire preuve d'une forme d'autorité.

Une autre problématique pourrait être nommée gestion de l'infobésité (c'est le terme québécois qui désigne la surabondance d'information). En effet, les élèves sont bombardés dans les médias d'informations biologiques qui ont une grande pertinence pour leur santé, leurs comportements en couple, en famille, en société comme citoyens ou consommateurs. Mais ils restent mal équipés de stratégies pour trier, valider, synthétiser, et produire des connaissances individuelles fondées scientifiquement (OCDE, 1999). Pourtant l'enseignement des sciences est souvent organisé autour de la présentation de savoirs dans des documents soigneusement simplifiés par le maître, prescrits et à apprendre exhaustivement (Jonassen, 1995). L'éducation aux sciences ne peut se dérober quant au développement de ces compétences d'intelligence informationnelle (Karsenti \& Dumouchel, 2010) si on veut rendre les élèves capables de trouver et d'utiliser les connaissances scientifiques pour comprendre les problèmes de leur monde et $\mathrm{y}$ agir de manière responsable.

L'autonomie dans le jugement qui valide les savoirs est donc un aspect crucial de la connaissance scientifique. En effet, la connaissance - forcément individuelle - ne peut être scientifique que dans la mesure où celui qui connaît peut 
établir et discuter les liens entre les données et les conclusions. Cette définition très ambitieuse implique qu'on ne connaît pleinement scientifiquement que très rarement. Elle indique cependant une direction pour l'action pédagogique: développer des connaissances scientifiques, c'est sevrer l'apprenant de la validation par le maître pour lui permettre de progressivement apprendre à l'assumer. C'est donc aider les élèves à trouver l'autorité de validation dans des expériences et des ressources ${ }^{1}$. Nous employons une définition de la connaissance scientifique qui permette de concevoir et de conduire les dispositifs, d'enseignement. Elle est donc pédagogique plus que philosophique ou épistémologique et s'appuie sur un processus de validation fondé sur des données, leur mise en contexte dans un paradigme et leur discussion (Osborne, 2010). Au niveau social, le processus de la science construit la validation des savoirs par l'observation et l'expérimentation, mais surtout par l'argumentation et le débat. Ainsi, une connaissance scientifique - bien que forcément personnelle - est éprouvée par un processus social de validation par la confrontation avec les pairs. Apprendre aux élèves à valider leurs connaissances dans la surabondance d'informations qui caractérise notre époque (Gibbons, 2000; Rocard et al., 2006), c'est contribuer à les rendre capables d'affronter la complexité du monde. Les processus de validation sont donc au cœur de la conception des dispositifs étudiés et de leur analyse.

\section{La surabondance d'information: un problème ou le début d'une solution?}

Sur un plan pédagogique, nous avons exploré des dispositifs visant à développer la capacité des élèves à valider eux-mêmes leurs connaissances scientifiques. Dans ce contexte, nous mettons en évidence que la surabondance d'information n'est pas forcément un problème pour l'école mais qu'elle peut être une opportunité puisqu'elle offre des ressources de qualités diverses dans lesquelles les élèves peuvent exercer leur capacité à valider. D'autre part, la nécessité d'assumer face aux autres la responsabilité d'une part du savoir est fondée sur les recherches sur l'apprentissage coopératif (Buchs, Gilles \& Butera, 2012; Mugny, Butera, Quiamzade, Dragulescu \& Tomei, 2003; Perret-Clermont, Grossen, Nicolet \& Schubauer-Leoni, 1996). Avec d'autres, ces fondements théoriques justifient le choix de l'investigation comme modèle pédagogique, et plus précisément une forme explicitement itérative, l'Inquiry Based Learning (IBL) (IBL Workshop Collective, 2001) qui développe la connaissance scientifique par une succession de phases de questionnement, d'investigation, de production écrite, de présentation et discussion, menant à une redéfinition des questions. La question y joue le rôle de principe structurant. Aussi le choix des questions que les élèves investiguent est crucial et pris dans une tension entre la nécessaire dévolution (que les élèves aient compris et aient envie de répondre à la question) et la responsabilité de l'enseignant concernant les objectifs et la couverture du programme. 
Comment définir alors les «bonnes» questions que les élèves puissent investiguer? Nous avons considéré que les bonnes questions sont celles du paradigme scientifique (Kuhn, 1972) courant. Or les écrits provenant de la recherche sont forcément structurés autour des questions et des réponses qu'on y apporte selon les modes de validation du paradigme. Il en résulte que les ressources conduisent d'autant plus vers les bonnes questions qu'elles sont proches de la production des savoirs et donc de l'origine du processus de transposition. Ainsi nous appellerons plus authentique un article dans la revue Nature - qui rapporte des expériences avec le contexte de production des résultats et le paradigme correspondant, la discussion et la mise en perspective - et moins authentique un article de vulgarisation dans Science \& Vie par exemple. Un livre scolaire ou un résumé produit par l'enseignant sont alors les moins authentiques. Ainsi la confrontation étayée et structurée - des élèves à des ressources d'authenticité croissante est un élément important des designs étudiés.

Notre projet a cherché à ancrer dans une profonde réflexion intégrant un large éventail de recherches pour les incarner dans des dispositifs d'enseignement assez radicaux éprouvés en classe sur l'année entière, pour analyser la construction de connaissances scientifiques par les élèves. Ainsi le projet s'est déroulé en trois phases: d'abord (2002-2010) nous avons développé un dispositif IBL, dans une posture de praticien réflexif (Schön, 1994), ensuite nous avons (2006-2012) procédé à la conceptualisation du design pour finalement identifier les variables critiques et définir les modalités d'une description en termes d'éléments de design (2009-2012). La thèse qui est l'aboutissement de ce projet (Lombard, 2012) ne se contente pas d'étudier un design particulier - forcément peu transférable à d'autres situations, ni de le comparer - d'ailleurs à quelle référence faudrait-il le comparer?

Son apport est de proposer une liste de recommandations éprouvées qui peuvent orienter le développement, la conduite de nombreux autres enseignements. Elle contribue aussi à la théorie en éducation en proposant un modèle synthétique d'IBL et met en évidence des variables permettant l'observation du développement des connaissances scientifiques chez les élèves.

La conceptualisation a porté sur un dispositif technopédagogique d'enseignement de la biologie par investigation qui a fonctionné plusieurs années au secondaire II. Il en est résulté notamment des conjectures issues de la recherche et liant des effets éducatifs attendus à des interventions et des variables de design. Ces conjectures incarnées (Sandoval, 2004) dans le dispositif sous forme d'éléments de design (ED) ont permis une description formelle du design. Ensuite, nous avons analysé plusieurs itérations du dispositif pour vérifier ces conjectures et tirer de cette analyse des recommandations de design ( $\mathrm{RD}$, avec leurs limites et de leurs combinaisons). Nous proposons ces RD pour faciliter la conception et le pilotage d'autres designs qui favoriseraient la construction de connaissances scientifiques dans des environnements infodenses.

Il a donc fallu préalablement développer un dispositif qui fonctionne et 
permette les apprentissages, trouver ou élaborer un cadre conceptuel capable de produire des instruments d'analyse les dimensions pertinentes du dispositif (Q0: Quelle conceptualisation du dispositif en permet une analyse pertinente?), puis décrire et valider le dispositif (Q1: comment réaliser un dispositif d'investigation à l'année assez efficace pour que les élèves d'année terminale y acquièrent des connaissances pertinentes aux yeux de l'institution?) et enfin l'analyser pour établir les liens entre des caractéristiques (leurs limites et leurs combinaisons) et des effets éducatifs conduisant à des connaissances scientifiques chez les élèves (Q2: comment conduire les élèves à se focaliser sur l'investigation scientifique dans un dispositif de type IBL?).

Nous allons principalement focaliser cet article sur la conceptualisation et la description du dispositif (Q0) et deux questions issues de la Q2. D'abord nous examinerons comment les élèves construisent des savoirs approfondis en biologie: comment l'investigation conduit-elle des questions vagues vers les questions précises et pertinentes au paradigme et en assure une réponse approfondie? puis nous adopterons le point du vue de l'enseignant et explorerons le guidage et la responsabilité Comment assurer le contrôle de l'investigation tout en respectant l'autonomie des élèves? Nous renvoyons le lecteur à la thèse (Lombard, 2012) disponible par l'archive ouverte de l'université de Genève pour le reste.

Pour des raisons éthiques, nous avons fait le choix de méthodes de recherche qui ne péjorent pas l'apprentissage. Nous avons retenu le paradigme DesignBased-Research (DBR) (Design Based Research Collective, 2003) qui propose des méthodes pour étudier comment les modifications d'un dispositif induisent des changements dans les effets éducatifs. Les caractéristiques critiques de cette approche sont d'affronter la complexité, d'intégrer la recherche dans des dispositifs d'enseignement, puis de conduire des investigations rigoureuses pour améliorer ces dispositifs et définir des recommandations de design.

Effectivement, au lieu de simplifier la complexité pour isoler quelques variables, on part de l'analyse de problèmes concrets sur le terrain par les chercheurs et les praticiens, on développe sur la base d'un cadre théorique des conjectures qu'on implémente dans des dispositifs, puis on conduit des investigations rigoureuses pour tester ces conjectures sur le terrain, on documente et on analyse pour produire des recommandations de design (Scardamalia \& Bereiter, 2006). Ces recommandations expriment des conjectures sur les effets éducatifs de chacun des éléments du design dont on a testé l'effet. Leur ensemble décrit les caractéristiques du dispositif qui se sont avérées importantes.

Cela conduit à des théories qui améliorent la compréhension des problématiques étudiées et qui peuvent être publiées et discutées (Brown, 1992; Collins, 1999; Design Based Research Collective, 2003). L'approche DBR s'applique à des interventions sur plusieurs années et permet de mettre en évidence des effets qui échappent souvent à la fenêtre d'observation (quelques heures ou semaines) de nombreuses recherches (Etkina, Karelina \& Ruibal-Villasenor, 2008; Songer, Lee, \& Kam, 2002). Par ailleurs la question de la pertinence à des situations de 
classe de résultats obtenus dans des conditions expérimentales bien contrôlées, inévitablement artificielles, reste débattue.

\section{Dispositif étudié et population}

L'étude a porté principalement sur 4 classes de biologie de niveau maturité soit 56 élèves de 19 ans environ.

Sept ans d'observation ont fourni les résultats à la Q0 soit les paramètres et variables permettant la description du dispositif. Leur construction et leur justification ne seront pas développées ici, mais nous en proposons une synthèse. Le dispositif est constitué en une communauté d'apprenants (Scardamalia, Bereiter, McLean, Swallow \& Woodruff, 1989) et organisé dans une structure coopérative (Buchs, Darnon, Quiamzade, Mugny \& Butera, 2008). L'investigation est structurée par des questions qui conduisent à des expériences, des recherches, la sélection par les élèves de ressources dans un environnement surabondant, la synthèse de réponses. Les activités d'écriture itérative et de discussion de ces écrits sont au cœur du dispositif de construction des connaissances: l'écriture qui soutient la construction de la pensée writing-to-learn et son débat en éprouve la solidité (Scardamalia \& Bereiter, 2006). Aussi, un espace d'écriture partagé (wiki) entre les élèves et l'enseignant étaye l'élaboration des connaissances.

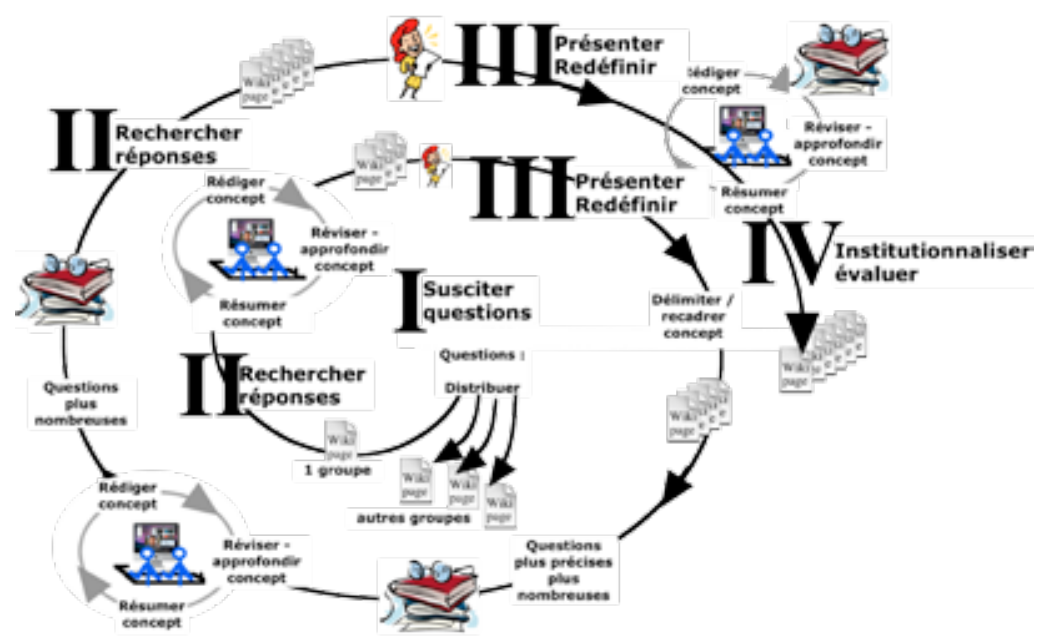

Figure 1: Le dispositif d'investigation parcourt plusieurs itérations d'un cycle synthétisé dans ce schéma: 1 Susciter des questions, les enregistrer, les organiser. 2 Rechercher des réponses, lire, comprendre, synthétiser, écrire. 3 Présenter aux pairs et redéfinir les questions. Ce cycle est répété et terminé par une phase 4: Institutionnalisation et évaluation de l'investigation. 
Les textes sont approfondis par de nombreuses révisions (typiquement 25-30), puis présentés aux pairs, ce qui conduit à des confrontations et des redéfinitions des questions. Les sujets traités ont été la génétique mendélienne et moléculaire, la biologie cellulaire et l'immunologie.

Chaque chapitre traité est divisé en 4 sous-thèmes, attribués à des groupes qui en sont responsables. Ils élaborent ainsi par groupes de trois ou quatre élèves une brochure sur laquelle ils s'appuient pour préparer leurs examens finaux.

C'est donc un document très important pour leur succès. Un cycle d'investigation dure 3-4 semaines, après quoi un autre chapitre est traité. Un cycle d'investigation est schématisé dans la Figure 1 et le Tableau 1 décrit les activités principales en regard des phases de l'investigation.

Nous présentons ci-dessous (cf. Tableau 1) une synthèse de ces descriptions et des phases que l'analyse a produites. Nous les avons structurées en fonction du déroulement et du pilotage des activités. Nous le proposons comme guide synoptique pour la gestion d'IBL dans l'éducation scientifique à la biologie.

\section{Tableau 1: Les activités, les phases du design abstrait et les variables pertinentes}

\begin{tabular}{|l|l|l|l|}
\hline Phase discernable & Activités & $\begin{array}{l}\text { Comportements et } \\
\text { activités cognitives } \\
\text { visées }\end{array}$ & Variables à observer \\
\hline Exploration & $\begin{array}{l}\text { I: Susciter des questions, } \\
\text { les enregistrer, les } \\
\text { organiser } \\
\text { II: Rechercher des } \\
\text { réponses, réviser, appro- } \\
\text { fondir concept }\end{array}$ & $\begin{array}{l}\text { Intérêt pour le thème } \\
\text { Questionnement } \\
\text { Repérage conceptuel } \\
\text { Premières définitions }\end{array}$ & $\begin{array}{l}\text { Appropriation par les } \\
\text { éèves des questions, } \\
\text { colonisation de tout le } \\
\text { champ conceptuel }\end{array}$ \\
\hline $\begin{array}{l}\text { Approfondissement } \\
\text { conceptuel }\end{array}$ & $\begin{array}{l}\text { II: Rechercher des } \\
\text { réponses, réviser, appro- } \\
\text { fondir concept } \\
\text { III: Échéance d'écriture, } \\
\text { consolidation, } \\
\text { évaluation, } \\
\text { IV: Présentation aux } \\
\text { pairs et redéfinition des } \\
\text { questions }\end{array}$ & $\begin{array}{l}\text { Affinage conceptuel } \\
\text { recherche, sélection, } \\
\text { synthèse } \\
\text { Confrontation intra- } \\
\text { texte, intertextes et avec } \\
\text { les ressources } \\
\text { Présentation aux pairs }\end{array}$ & $\begin{array}{l}\text { Délimitation concep- } \\
\text { tuelle } \\
\text { Complexité épistémique } \\
\text { s'accroissant }\end{array}$ \\
\hline $\begin{array}{l}\text { Institutionnali- } \\
\text { sation }\end{array}$ & $\begin{array}{l}\text { IV: Présentation aux } \\
\text { pairs et redéfinition des } \\
\text { questions } \\
\text { V: Institutionnalisation } \\
\text { et évaluation }\end{array}$ & $\begin{array}{l}\text { Stabilisation et défini- } \\
\text { tions de la terminologie } \\
\text { Explication des concepts } \\
\text { Révision de la structure } \\
\text { Révision en vue } \\
\text { diffusion pour la classe. }\end{array}$ & $\begin{array}{l}\text { Structure et définitions } \\
\text { Approfondissement } \\
\text { (complexité épisté- } \\
\text { mique) } \\
\text { Limites temporelles de } \\
\text { l'institution }\end{array}$ \\
\hline
\end{tabular}

Nous renvoyons le lecteur à la thèse pour la justification et une description très détaillée du dispositif en termes d'individus, groupes, activités, rôles et ressources (Kobbe, 2005). 


\section{Données et méthodologies}

Le fait que le chercheur soit en même temps l'enseignant a permis d'explorer un design franchement radical et d'accéder à des mesures de pertinence accrue (Barab, 2006), mais implique une grande prudence dans le choix des données (indépendantes de l'enseignant), et leur interprétation.

Une part importante des données provient des enregistrements automatiques par le serveur wiki de toutes les versions successives d'un même texte que les élèves produisent, aussi bien en classe qu'à partir du domicile (pour chaque document de groupe une centaine d'enregistrements, 145 documents soit au total un corpus de près de 10 millions de mots). La recherche a donc accès à une partie de l'activité scolaire des élèves en dehors de l'école, qui échapperait à une observation en classe seulement. Le nombre de mots, le nombre de questions, leur filiation conceptuelle ont été établis pour chaque révision du texte (25-30 typiquement).

Pour mesurer la progression de l'approfondissement, nous avons mesuré la complexité épistémique (CE) des réponses selon une échelle pertinente pour la biologie qui se réfere à Hakkarainen (2003) et adaptée par Zhang, Scardamalia, Lamon, Messina et Reeve (2007 p.143). Les textes ont été découpés en unités de sens qui ont été codées sur cette échelle à 4 niveaux $(1=$ descriptions simples, 2 = descriptions étayées, 3 = explications simples, $4=$ explications étayées) selon les critères de ces auteurs. Nous avons mesuré la CE d'un échantillonnage de documents arbitraire: le même sous-thème (Immunité humorale) à la même période, sur quatre années (2006-2010). Nous avons aussi comparé le document produit en début d'année et en fin d'année par le même groupe. Chaque document a été codé (simple codage) à trois moments représentatifs des phases établies sur la base des mesures de nombre de questions. La filiation des questions a été établie en comparant les enregistrements successifs sur la base de la position dans le texte et des liens logiques.

Des questionnaires ont été administrés aux élèves en fin d'année. Un questionnaire par e-mail a été envoyé aux étudiants environ un an après. Ces questionnaires portaient sur leurs perceptions de la qualité des apprentissages, leur pertinence à leurs études et sur l'efficacité des stratégies développées. D'autres sources (journal d'observateurs en classe, notamment) ont permis une triangulation des données.

Globalement l'approche méthodologique fait appel à un éventail de méthodes adaptées aux questions et aux données à étudier concurrent mixed methods design (Tashakkori \& Teddlie, 2003). Il s'agit d'une triangulation qui vise «à augmenter la pertinence [...] par la prise en compte de la complexité du terrain...» (Paquay, Crahay \& De Ketele, 2006). 


\section{Quelques résultats choisis}

Dans le paradigme DBR, il n'est pas question de comparer un dispositif à une référence, ni d'établir l'efficacité générale des modèles d'investigation. Cependant, il faut préalablement montrer que le design qu'on fait évoluer est suffisamment efficace et adapté au terrain scolaire pour que l'analyse des effets des conjectures incarnées (les 27 Recommandations de Design = RD) y soit pertinente. C'est cette analyse des $\mathrm{RD}$ et de leurs effets qui est l'objet de recherche.

Ainsi le dispositif a été validé par les résultats aux examens finaux de maturité, et un expert a évalué les savoirs produits par les élèves: ils ont été jugés satisfaisants à très bons selon les groupes échantillonnés et correspondant bien au curriculum. Les réponses au questionnaire post-secondaire indiquent que $89 \%$ des étudiants considèrent avoir été «... bien préparé pour vos études» et $11 \%$ indiquent des réserves mineures $(\mathrm{N}=18)$. Avec d'autres données nous avons pu ainsi estimer que ce dispositif fournit un terrain d'exploration crédible pour nos questions de recherche.

Un des résultats qui ressort très clairement est l'interdépendance des RD, aussi une présentation incomplète est forcément discutable. En effet, nos résultats suggèrent qu'une prise en compte des paramètres principaux est plus importante que le degré de perfection atteinte sur chacun des paramètres. Cette présentation focalisée sur quelques paramètres gagne en clarté mais perd une partie de la solidité que le croisement des données justifie dans le document complet (Lombard, 2012).

Nous avons chois de formuler les résultats en termes de définition du milieu (au sens ou (Brousseau, 1990) le définit) auquel l'élève est confronté et présenterons une partie des sous-questions de la Q2 Comment l'investigation conduit-elle des questions vagues vers les questions précises et pertinentes au paradigme et en assure une réponse approfondie?

Les conjectures que nous avons vérifiées sont notamment:

RD6: Laisser les «bonnes questions» émerger de la confrontation aux ressources authentiques dans le paradigme, susciter cette confrontation si nécessaire.

RD7: La confrontation à des ressources authentiques conduit l'investigation vers le paradigme dont sont issues ces ressources.

RD10: Les réponses doivent traiter le concept défini par la question: unicité conceptuelle des questions et des réponses correspondantes.

RD14: L'enseignant doit intervenir pour qu'une question au moins colonise chaque secteur important du champ conceptuel et évite les zones d'ombre conceptuelle.

RD15: L'effet centripète peut assurer que des questions mal posées se développent vers les bonnes questions du paradigme. 
Parmi les résultats confirmant ces conjectures, relevons ici l'analyse des questions (Cf. Figure 2) qui montre comment, à partir d'une question initiale, de nouvelles questions apparaissent et divergent, et comment certaines sont parfois reformulées au cours de l'investigation. Le fait que l'investigation conduise depuis une question de départ vers de nombreuses questions plus précises y est manifeste. Un exemple détaillé de filiation des questions en Figure 3 montre comment le dispositif conduit une question vague à être subdivisée en cinq nouvelles, puis trois sont reformulées ou subdivisées. L'affinage conceptuel est manifesté par la précision accrue des questions et leur convergence vers les concepts structurants: notamment la question «Les lymphocytes B peuvent-ils fonctionner sans les lymphocytes T?» s'approche de la question des interactions entre lymphocytes $\mathrm{B}$ et $\mathrm{T}-$ les concepts centraux du champ conceptuel. L'analyse des réponses et leur affinage contribue avec d'autres résultats à confirmer la RD10: la simple exigence que les réponses traitent effectivement de la question conduit à l'affinage conceptuel et oblige l'investigation à coller aux questions, assurant l'efficacité du pilotage (elle évite la dilution de la question et le risque de dérive vers la facilité d'accès des ressources).

La RD15 est confirmée par l'observation de l'affinage des questions: on observe qu'elles se développent par des chemins différents chaque année, mais convergent toujours vers les questions centrales structurant le domaine (Wiggins $\&$ McTighe, 2000). Le rapport de l'expert confirme que les concepts importants du curriculum ont bien été traités. Ce qui est intéressant est que les questions se sont non seulement raffinées, mais ont convergé vers les questions pertinentes du paradigme de la biologie (les mécanismes sous-jacents) le plus souvent par la confrontation à des ressources plus authentiques (au sens de moins scolaire, plus près de la recherche comme décrit plus haut). L'examen des textes et des interventions de l'enseignant montre que la convergence se fait vers les concepts du paradigme d'où les ressources proviennent et qu'un accès facilité et un feed-back qui renvoie vers ces ressources authentiques suffisent le plus souvent à permettre ce que nous appelons «l'effet centripète conceptuel». En somme, l'authenticité des ressources suscite les bonnes questions et l'approfondissement dans le paradigme. Ainsi en supervisant seulement l'affinage des questions, sans imposer ou sélectionner les ressources (l'accès libre à internet est ouvert), l'enseignant vérifie que l'investigation progresse vers les concepts structurants du paradigme. En effet les questions déterminent le type de ressource que les élèves trouveront et celles-ci déterminent les questions auxquelles l'approfondissement conceptuel conduira dans l'étape suivante d'investigation. Cependant les feed-back de l'enseignant ne corrigent pas mais renvoient à des ressources d'authenticité croissante (RD23 discutée plus bas). D'autre part, quand une part importante du champ conceptuel n'est pas colonisée par une question au moins l'enseignant doit susciter une question (RD14). 


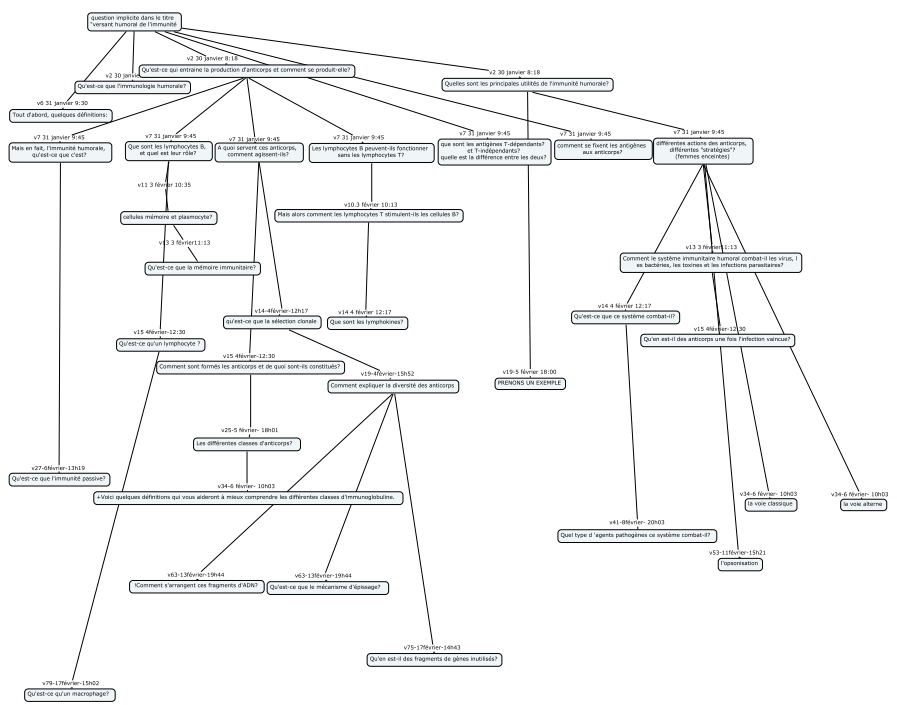

Figure 2: Vue d'ensemble du suivi des questions d'un groupe d'investigation, le temps se déroule de haute en bas. Sans permettre la lecture détaillée des questions (cf. plus bas pour un agrandissement), on peut y voir l'affinage conceptuel, qui se traduit par l'arborescence des question que les élèves poursuivent. Une question est subdivisée en plusieurs nouvelles questions au fur et à mesure que les élèves approfondissent.

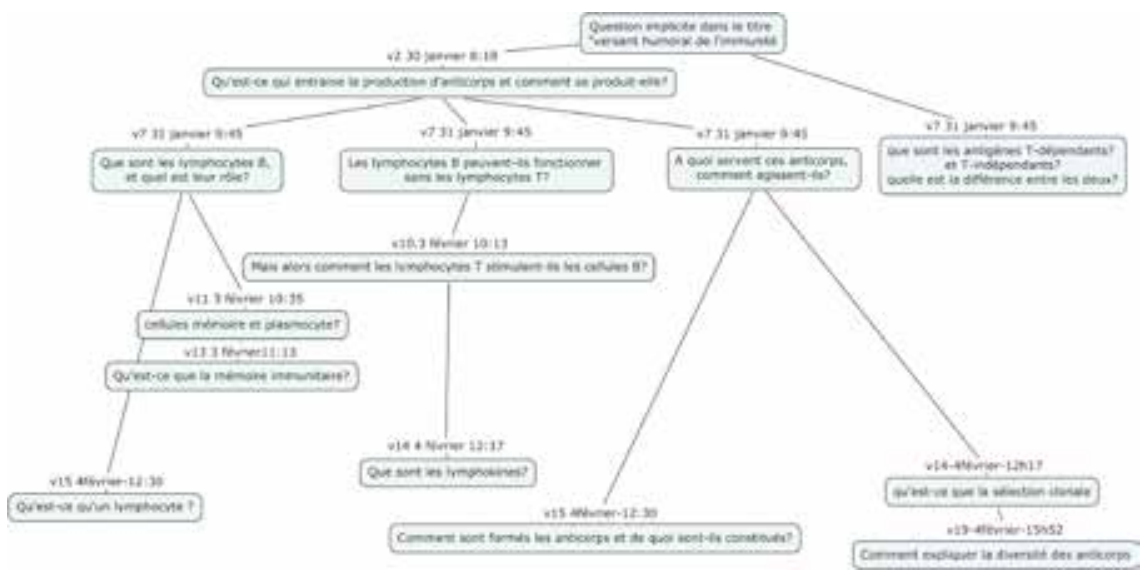

Figure 3: Un exemple détaillé de filiation des questions: une question vague est subdivisée en cinq nouvelles puis trois sont reformulées ou subdivisées. L'affinage conceptuel est manifesté par la précision accrue des questions et leur convergence vers les concepts structurants: notamment la question des interactions entre lymphocytes $B$ et $T$ qui est au centre du domaine concerné. 


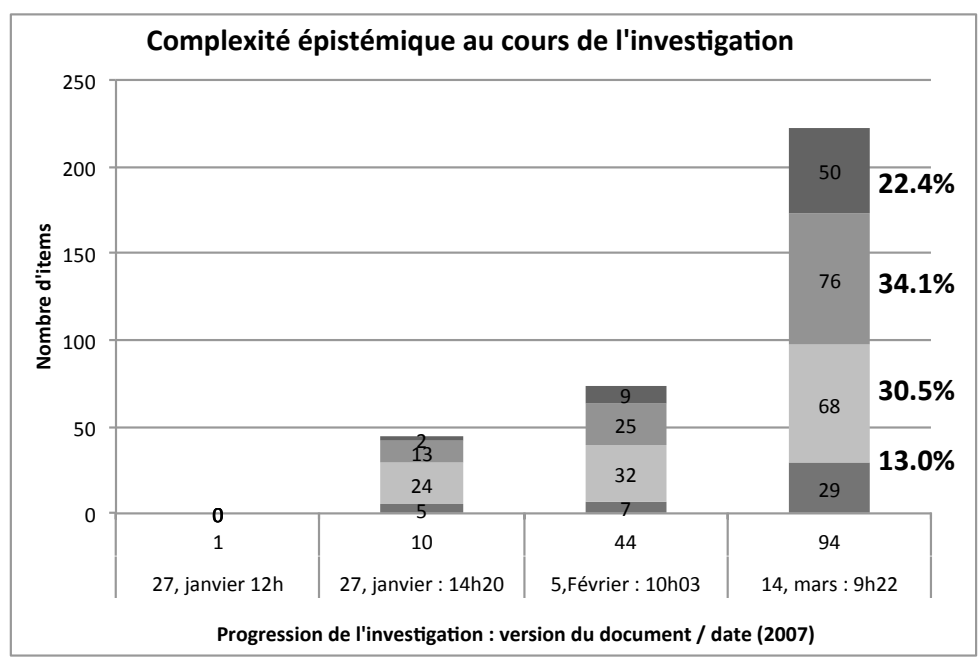

Figure 4: Complexité épistémique d'une investigation pour un groupe en fonction du temps, avec indication des pourcentages. La complexité épistémique des réponses dans un document wiki augmente au cours de son élaboration (fin d'année 2007).

Nous allons ici présenter quelques résultats concernant la nature des interventions de l'enseignant et notamment: comment assurer le contrôle de l'investigation tout en respectant l'autonomie des élèves?

Nous avons vu que, pour des raisons scientifiques et propres à la pédagogie IBL, une séparation claire de l'autorité scientifique (de validation) et pédagogique était souhaitable, que la validation de leurs connaissances doit être trouvée par les élèves dans la confrontation à des ressources (plus) authentiques. L'intervention de l'enseignant doit donc être pensée et effectuée principalement en modifiant le milieu. Elle peut prendre la forme d'un étayage structurant - qui encadre et organise, rassure et guide - mais doit aussi prendre la forme d'un étayage stimulant - qui oblige les élèves à voir et à résoudre les incohérences (Reiser, 2004) dans leurs productions (intra-documents, inter-documents et avec des ressources externes). En particulier, l'intervention de l'enseignant dans les textes doit favoriser le conflit socio-cognitif et créer un climat qui développe notamment l'interdépendance positive et la responsabilité individuelle (Buchs et al., 2012; Johnson \& Johnson, 1989). Cette posture implique de fournir une ressource judicieusement choisie pour permettre à l'élève de progresser plutôt que de simplement "corriger" les erreurs - ce qui rendrait impossible le processus de validation par l'élève. Cela nécessite la gestion d'un considérable stock de ressources (plus de mille références dans des répertoires thématiques et seize mille images) qui puissent être aisément indiquées aux élèves dans l'espace d'écriture wiki partagé. 
RDA3: Transférer aux ressources la validation scientifique des connaissances, conserver l'autorité pédagogique

RD4: Dévoluer progressivement la validation des savoirs recherchés

RD8: L'investigation est pilotée par des questions négociées en rapport avec les objectifs

RD16: La transition des activités peut être pilotée en observant la colonisation du champ conceptuel et l'émergence de la complexité épistémique

RD23: Le feed-back de l'enseignant vise à créer des conflits cognitifs en relevant des incohérences ou des différences d'explication intra-texte, inter-textes et avec des ressources externes

RD26: Les apprenants sont confrontés à des ressources très nombreuses et de qualité hétérogène

La principale confirmation de ces $\mathrm{RD}$ est que le curriculum est effectivement couvert, les objectifs atteints, les résultats aux examens et les perceptions de la qualité de leurs apprentissages (questionnaire post-secondaire) satisfaisants. La RD8 mérite quelques commentaires: elle est dépendante de la RD10 (unicité conceptuelle des questions et réponses) qui assure que les questions guident effectivement l'investigation. La supervision par l'enseignant est nécessaire (RD16), mais l'essentiel de l'évolution des questions est déterminé par la confrontation à des ressources authentiques. C'est-à-dire que l'utilisation d'une ressource pour répondre à une question suscite forcément de nouvelles questions découlant de la compréhension qui s'approfondit (c'est l'affinage conceptuel manifeste sur la Figure 2). Ce qui est très intéressant est que ces nouvelles questions reflètent le paradigme des ressources employées: en d'autres termes les nouvelles questions sont les «bonnes» questions. Ainsi le choix des ressources auxquelles les élèves sont confrontés détermine les questions qu'ils vont poursuivre après l'avoir synthétisé. Ainsi le guidage de l'enseignant ne s'effectue pas tant par la prescription des questions, mais surtout en veillant à ce que les élèves soient confrontés à des ressources qui contiennent les «bonnes» questions inscrites dans leur organisation conceptuelle. Comme les feed-back de l'enseignant orientent vers ces ressources parce qu'on y trouve des réponses difficiles à trouver ailleurs, ces ressources sont assez naturellement intégrées dès que les élèves peuvent en profiter. Le pilotage par le milieu auquel les élèves sont confrontés se manifeste ici en laissant le laisser paradigme de la biologie s'imposer aux élèves par les ressources. De plus l'autorité scientifique y est explicitement trouvée et le sevrage de l'autorité de validation du maître en découle.

Une autre facette de l'activité de l'enseignant et la prescription des activités décrites au Tableau 1: le moment où elles doivent intervenir est critique. Il s'agit de planification en termes d'étapes de la progression conceptuelle des élèves (Altet, 1993). Nous avons montré que la complexité épistémique est une bonne mesure de cette progression, mais qu'elle n'est apparente dans l'espace d'écriture 
partagé que suite aux activités prescrites. Par contre le nombre de questions et leur nature révèlent bien l'état de cette progression. Notamment la colonisation (RD16) du champ conceptuel: l'enseignant doit s'assurer que parmi les questions nombreuses de la phase d'exploration - même vagues et encore mal formulées il y en ait au moins une dans chaque partie du champ conceptuel, et intervenir si nécessaire pour susciter des questions (proposer un texte, monter une incohérence, relever un implicite) qui colonisent une éventuelle zone d'ombre. Un exemple est décrit dans la thèse. Ainsi l'enseignant ne fixe pas les activités prévues que les étapes conceptuelles que les élèves doivent atteindre à des moments déterminés. Cela demande une souplesse dans les interventions pour guider les élèves dans des cheminements conceptuels très variés et une organisation permettant d'aiguiller les élèves très rapidement parmi des ressources très nombreuses. Un vaste système de gestion de documents digitalisés permet de référencer dans l'espace d'écriture la ressource susceptible de faire progresser l'élève.

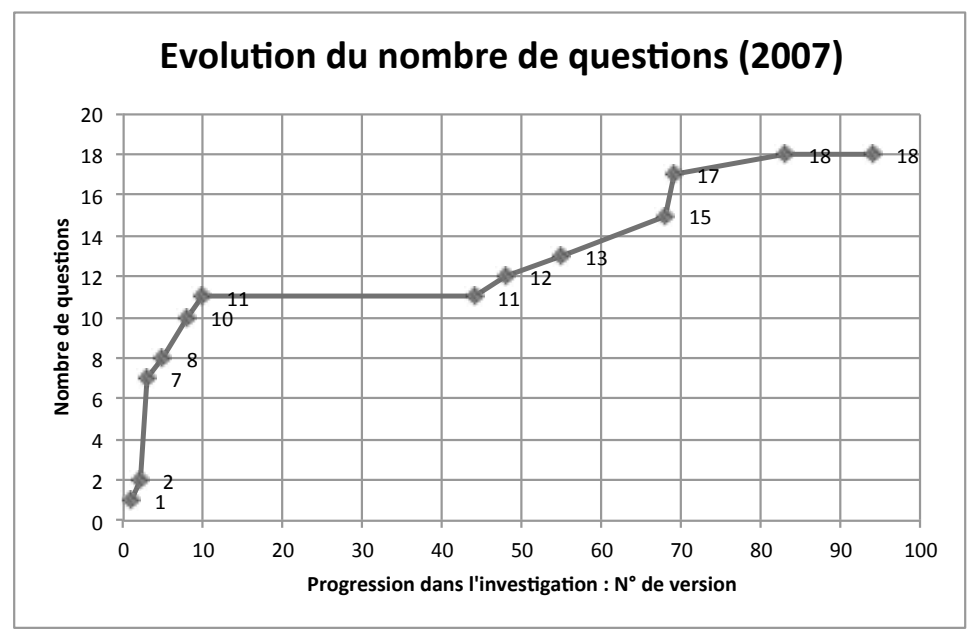

Figure 5: L'évolution du nombre de questions montre bien les phases distinctes et l'effet des interventions de l'enseignant qui prescrit les activités (flèches verticales).

Nous pourrions synthétiser ces résultats sous forme de quatre conclusions:

1 L'autonomie dans la validation de leurs connaissances par les élèves ouvre la porte à la connaissance scientifique.

2 La dissociation de l'autorité pédagogique et scientifique permet le guidage sans compromettre l'autonomie de validation.

3 L'affinage des questions conduit l'affinage conceptuel pour autant que la règle d'unicité conceptuelle des questions et réponse soit appliquée.

4 L'authenticité des ressources auxquelles les élèves sont confrontés suscite les bonnes questions. 


\section{Conclusion: Une description abstraite qui illustre l'interdépendance des recommandations et des fonde- ments théoriques}

En synthétisant cette analyse, nous avons produit une description abstraite du design IBL (on pourrait dire le Modèle sous-jacent) en termes de milieu (Brousseau, 1990) plutôt que d'action enseignante et réussi à définir l'IBL par seulement trois recommandations fondamentales d'attitudes basées sur trois fondements pédagogiques. Leur interdépendance nous a conduit à représenter le design abstrait par une structure hexagonale (cf. Figure 2) qui illustre bien les dépendances et causalités circulaires. Les recommandations les plus cruciales concernent les attitudes visées chez les élèves (RDA). Chacune de ces trois recommandations d'attitudes RDA1 à RDA3 synthétise une ou plusieurs conjectures issues de l'analyse de nos résultats.

RDA1: Susciter la responsabilité par les élèves d'une partie des savoirs face aux pairs

RDA2: Susciter l'adhésion à un but partagé d'approfondissement conceptuel en biologie

RDA3: Transférer aux ressources la validation scientifique des connaissances, conserver l'autorité pédagogique

Chacune des ces trois recommandations de design d'attitudes RDA s'appuie sur deux des fondements pédagogiques: i) Communauté apprenante qui détermine RDA1 et RDA2, ii) Structure coopérative qui détermine RDA1 et RDA3, iii) Authenticité dans le paradigme qui détermine RDA3 et RDA2. L'ensemble de ces concepts dépendants les uns des autres forme une structure cohérente qui conduit à des connaissances scientifiques illustrées au centre. Nous avons mis en évidence trois aspects de la production de connaissances scientifiques et les relions aux RDA: l'approfondissement conceptuel dans le paradigme résulte de la RDA2: l'adhésion à un but partagé d'approfondissement en biologie, l'implication dans l'apprentissage découle de la responsabilité face aux pairs d'une part des savoirs (RDA1), et l'autonomie dans la validation scientifique découle de la RDA3: dévolution de la validation scientifique. La connaissance scientifique résulte de l'effet - convergeant au centre - de ces recommandations (cf. Figure 2).

Au-delà de cette structure abstraite, les apports qui nous semblent se démarquer le plus de la littérature et pouvoir prétendre à une certaine originalité sont:

- La séparation claire entre autorité pédagogique et scientifique qui aide à résoudre le paradoxe du guidage malgré la dévolution et permet le développement de connaissances scientifiquement validées.

- Le pilotage dans l'espace conceptuel par les questions négociées dans le cadre des objectifs. 


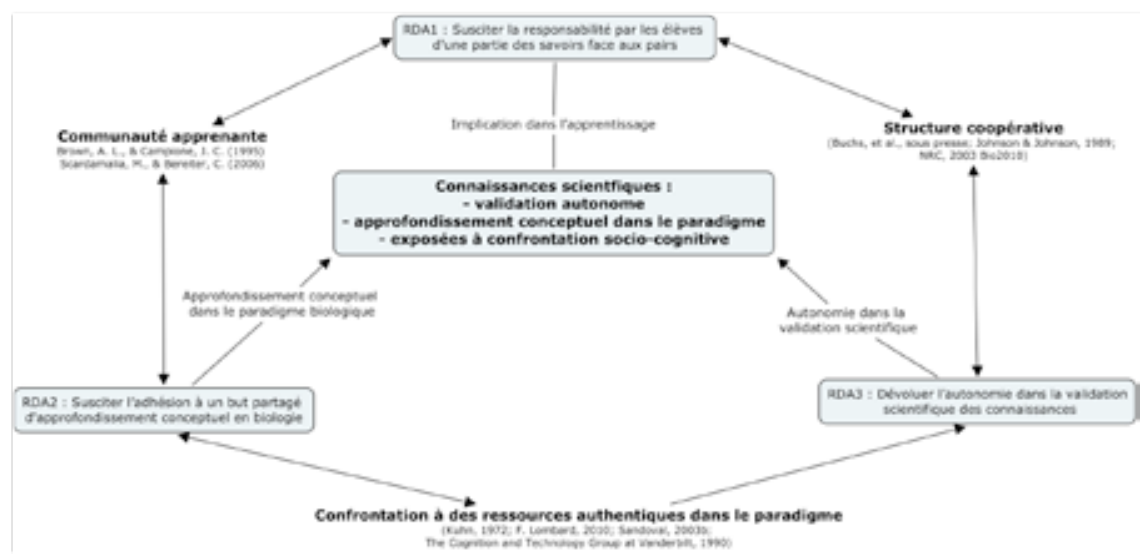

Figure 6: Structure abstraite de designs visant le développement de connaissances scientifiques. Elle construit dans des activités de co-écriture trois aspects des connaissances scientifiques à partir des trois principales recommandations de design d'attitudes (RDA). Chacune des RDA est fondée par les deux fondements pédagogiques la jouxtant. Dans ce schéma de synthèse, les activités d'écriture partagée qui soutiennent cette construction de la connaissance ne sont pas explicitement figurées.

- L'affinage conceptuel guidé par l'interaction entre les questions et les ressources. i) La règle d'unicité des questions-réponses qui conduit à l'affinage conceptuel (concept refinement et subsumption) et oblige l'investigation à coller aux questions, assurant l'efficacité du pilotage (évite la dilution et la dérive vers la facilité d'accès des ressources); ii) l'adéquation paradigmatique obtenue par la confrontation progressive aux ressources authentiques qui induisent les questions du paradigme; iii) la structure du champ conceptuel qui oriente vers les concepts structurants, que nous proposons de nommer: force centripète conceptuelle; iv) l'infobésité comme une solution: l'hétérogénéité de la qualité des ressources affrontées par l'élève donne du sens à la validation. L'accès à internet expose justement à des documents incertains voire mauvais, mais aussi à des ressources authentiques dans le paradigme.

- L'identification de phases observables dans l'IBL et de variables permettant le pilotage conceptuel de ces phases d'investigation (colonisation de tous les domaines du champ conceptuel, montée de la complexité épistémique).

- L'étayage de l'investigation dans un espace d'écriture: la production en commun par les élèves d'un document décisif pour leurs examens.

- La durée nécessaire à l'investigation pour porter ses fruits: i) la durée pour élaborer un contrat didactique d'IBL et développer chez les élèves les compétences d'intelligence informationnelle qui produisent des connaissances approfondies (plusieurs mois); ii) la durée de l'investigation pour que les élèves produisent des connaissances approfondies, mesurée par la complexité épistémique (3-4 semaines). 
La pertinence des recommandations proposées dans ce dispositif y a été établie, mais leur transférabilité à d'autres contextes n'est pas automatique et nous en proposons une discussion dans la thèse. Au terme de cette recherche, nous avons évoqué quelques perspectives qu'elle ouvre. Nous proposons la complexité épistémique comme mesure pertinente de la progression conceptuelle, comme mesure des apprentissages indépendante du contenu qui pourrait permettre la comparaison de dispositifs ou d'interventions. Il pourrait être intéressant d'en développer une version simplifiée qui puisse être utilisée par les enseignants comme mesure de la progression conceptuelle, de l'adéquation aux objectifs, ... Nous aimerions analyser - notre corpus de données sur l'évolution des questions (toutes les versions enregistrées soit près de 10 millions de mots) le permettrait les cheminements conceptuels pour confirmer l'effet centripète conceptuel dans d'autres sous-thèmes.

Nous pensons que développer un formalisme de description de designs en termes de RD permettrait des comparaisons de modèles pédagogiques, une formation des enseignants et des choix dans le pilotage des enseignements de biologie plus pertinents.

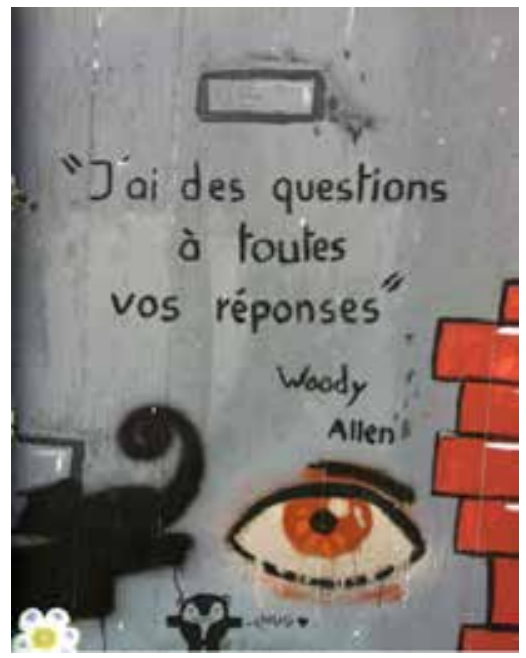

\section{Palissade au Collège Calvin, été 2012}

Normalement, maintenant, plus l'ouvrage est gros et détaillé, plus cela me conforte, car j'en suis presque sûr que j'y trouverai les réponses.

La seule difficulté est de comprendre profondément ce qu'on recherche. Après, il n'est pas difficile de trouver si on est sûr de notre objectif

Commentaires d'élèves dans le questionnaire de fin d'année. 


\section{Note}

1 Certaines ressources relatant des expériences peuvent être considérées sur le plan pédagogique comme des expériences par procuration parce qu'elles permettent à l'élève de développer au moins un peu sa capacité à utiliser des données scientifiques, à les discuter et les mettre en perspective

\section{Références}

Altet, M. (1993). Préparation et planification. In J. Houssaye (Éd.), La Pédagogie, une encyclopédie pour aujourd'hui (pp. 78-88). Paris: ESF.

Barab, S. (2006). Design-Based Research. A Methodological Toolkit for the Learning Scientist. In R. K. Sawyer (Éd.), The Cambridge Handbook Of The Learning Sciences (pp. 153-169). New York: Cambridge University Press.

Brousseau, G. (1990). Le contrat didactique: le milieu. Recherches en didactique des mathématiques, 9(9.3), 309-336.

Brown, A. L. (1992). Design Experiments: Theoretical and Methodological Challenges in Creating Complex Interventions in Classroom Settings. The Journal of the Learning Sciences, 2(2), 141-178.

Buchs, C., Darnon, C., Quiamzade, A., Mugny, G. \& Butera, F. (2008). Conflits et apprentissage. Régulation des conflits sociocognitifs et apprentissage. Revue française de pédagogie, 2, 105-125.

Buchs, C., Gilles, I. \& Butera, F. (2012). Optimiser les interactions sociales lors d'un travail de groupe grâce à l'apprentissage coopératif. In E. Bourgeois \& G. Chapelle (Éd.), Apprendre et faire apprendre. Paris: Presses Universitaires de France.

Collins, A. (1999). The changing infrastructure of education research. Issues in education research, 289-198.

Design Based Research Collective (2003). Design-Based Research: An Emerging Paradigm for Educational Inquiry. Educational Researcher, 32(1), 5-8.

Etkina, E., Karelina, A. \& Ruibal-Villasenor, M. (2008). How long does it take? A study of student acquisition of scientific abilities. Physical Review Special Topics - Physics Education Research, 4(2), 020108.

Gibbons, M. (2000). Mode 2 society and the emergence of context-sensitive science. Science and Public Policy, 27(3), 159-163.

Hakkarainen, K. (2003). Progressive inquiry in a computer-supported biology class. Journal of Research in Science Teaching, 40(10), 1072-1088.

IBL Workshop Collective. (2001, February 21, 2001). Our definition of Inquiry. Inquiry Teaching \& Learning Workshop A Workshop for Educators, Librarians and Faculty in Teacher Education Programs, from http://inquiry.uiuc.edu/inquiry/definition.php3

Johnson, D. W. \& Johnson, R. T. (1989). Cooperation and Competition: Theory and Research. Edina, MN: Interaction Book Company.

Jonassen, D. (1995). Constructivism and Computer-Mediated Communication in Distance Education. American Journal of Distance Education, 9(2), p7-26.

Karsenti, T. \& Dumouchel, G. (2010). Former à la compétence informationnelle: une nécessité pour les enseignants actuels et futurs. In T. Karsenti \& G. Dumouchel (Éd.), Le développement de l'intelligence informationnelle (pp. 189-213): ASTED.

Kirschner, P. A., Sweller, J. \& Clark, R. E. (2006). Why Minimal Guidance During Instruction Does Not Work: An Analysis of the Failure of Constructivist, Discovery, Problem-Based, Experiential, and Inquiry-Based Teaching. Educational Psychologist, 41(2), 75-86. doi: doi:10.1207/s15326985ep4102_1

Kobbe, L. (2005). Framework on multiple goal dimensions for computer-supported scripts. Kaleidoscope (Vol. D29.2.1): Knowledge Media Research Center (KRMC). 
Kuhn, T. S. (1972). La structure des révolutions scientifiques. Paris: Flammarion.

Lombard, F. (2012). Conception et analyse de dispositifs d'investigation en biologie: comment conjuguer autonomie dans la validation scientifique, approfondissement conceptuel dans le paradigme et couverture curriculaire ? Doctorat, Université de Genève, Genève. Retrieved from http://archive-ouverte.unige.ch/vital/access/manager/ Repository/unige:24656

Mugny, G., Butera, F., Quiamzade, A., Dragulescu, A. \& Tomei, A. (2003). Comparaisons sociales des compétences et dynamiques d'influence sociale dans les tâches d'aptitudes. L'Annéee psychologique, 103(3), 469-496.

OCDE. (1999). PISA. Mesurer les connaissances et compétences des élèves - un nouveau cadre d'évaluation. Paris: OCDE.

Osborne, J. (2010). Arguing to Learn in Science: The Role of Collaborative, Critical Discourse. Science, 328(5977), 463-466. doi: 10.1126/science.1183944

Paquay, L., Crahay, M. \& De Ketele, J. M. (2006). L'analyse qualitative en éducation: Des pratiques de Recherche Aux Critères de Qualité: Hommage à Michael Huberban De Boeck Université. Huberman. Bruxelles: De Boeck Université.

Perret-Clermont, A.-N., Grossen, M., Nicolet, M. \& Schubauer-Leoni, M.-L. (1996). La construction de l'intelligence dans l'interaction sociale. Berne, Suisse: Peter Lang.

Popper, K. R., Lorenz, K., Sexl, R. U., Kreuzer, F., \& Etoré, J. (1990). L'avenir est ouvert: Entretien d'Altenberg: textes du symposium Popper à Vienne. Vienne, Autriche. p. 63

Reiser, B. J. (2004). Scaffolding complex learning: The mechanisms of structuring and problematizing student work. The Journal of the Learning Sciences, 13(3), 273-304.

Rocard, M., Csermely, P., Jorde, D., Lenzen, D., Walberg-Henriksson, H. \& Hemmo, V. (2006). Science education now: A renewed pedagoy for the future of Europe. In M. Rocard (Ed.), Directorate-General for Research. Bruxelles: OECD EUROPEAN COMMISSION.

Sandoval, W. A. (2004). Developing Learning Theory by Refining Conjectures Embodied in Educational Designs. Educational Psychologist, 39(4), 213-223.

Scardamalia, M. \& Bereiter, C. (2006). Knowledge building: Theory, pedagogy, and technology. In K. Sawyer (Éd.), The Cambridge handbook of the learning sciences (pp. 97-115). New York: Cambridge University Press.

Scardamalia, M., Bereiter, C., McLean, R. S., Swallow, J. \& Woodruff, E. (1989). Computer-supported intentional learning environments. Journal of Educational Computing Research, 5(1), 51-68.

Schön, D. A. (1994). Le praticien réflexif, à la recherche du savoir caché dans l'agir professionnel. Montréal: Logiques.

Songer, N. B., Lee, H. S. \& Kam, R. (2002). Technology-rich inquiry science in urban classrooms: What are the barriers to inquiry pedagogy? Journal of Research in Science Teaching, 39(2), 128-150.

Tashakkori, A. \& Teddlie, C. (2003). Handbook of mixed methods in social \& behavioral research. Sage Publications, Inc. Tousand Oaks, California, USA

Tobias, S. \& Duffy, T. (2009). Constructivist instruction: success or failure? Taylor \& Francis. New York: Routledge.

Wiggins, G. \& McTighe, J. (2000). Understanding by Design. Upper Saddle River, NJ: Prentice Hall.

Zhang, J., Scardamalia, M., Lamon, M., Messina, R. \& Reeve, R. (2007). Socio-cognitive dynamics of knowledge building in the work of 9-and 10-year-olds. Educational Technology Research and Development, 55(2), 117-145.

Mots-clés: Enseignement de la biologie, enseignement secondaire, dispositif d'investigation, écriture partagée 


\section{Konzeption und Analyse problemzentrierten Lernens im Biologieunterricht: Wie können die autonome Nutzung validierter, naturwissenschaftlicher Grundlagen, die konzeptionelle Vertiefung und die Einhaltung des Lehrplans verbunden werden?}

\section{Zusammenfassung}

Das pädagogische Modell «Inquiry Learning» wird im Rahmen von EU-Bildungsprogrammen propagiert. In Frankreich ist es für den naturwissenschaftlichen Unterricht auf der Sekundarstufe II vorgeschrieben. Im Zentrum des Modells stehen die Schülerinnen und Schüler, die aufgefordert sind, ihre eigenen Fragestellungen zu formulieren. Diese Verlagerung von der Lehrerzentrierung zur Schülerzentrierung schafft eine Spannung, wenn es darum geht, Basiswissen gründlich zu vermitteln und den Lehrplan einzuhalten.

Wir entwarfen in den letzten 10 Jahren ein "Inquiry Learning-Design" für den Biologieunterricht auf der Sekundarstufe II. Wir analysierten Daten von vier Jahren, um eine Konzeptualisierung, eine abstrakte Beschreibung und relevante Variablen (z.B. epistemische Komplexität) zu extrahieren. Im Kontext eines Design-Based Research-Ansatzes analysierten wir die Entstehung schriftlicher Arbeiten der Schülerinnen und Schüler mittels Log-Datei der Schreibplattform. Wir führten Fragebogenerhebungen sowohl am Ende der Sekundarstufe II als auch ein Jahr nach dem Übertitt an die Universität durch. Unser Inquiry Learning-Modell kann in 27 Designregeln beschrieben werden. Wir schlagen insbesondere vor, didaktische und wissenschaftliche Aspekte zu trennen. Eine Synthese des Modells kann mit einer Interdependenz von drei Variablen definiert werden: i) die Verantwortung, einen Teil des Wissens zu produzieren, muss von jeder Schülerin und jedem Schüler angenommen werden, ii) es muss ein gemeinsames Ziel sein, Wissen zu vertiefen, und iii) es sind authentische naturwissenschaftliche Quellen für die konzeptionelle Ausgestaltung der Fragen zu nutzen.

Schlagworte: Biologieunterricht, problemzentriertes Lernen, Schreibplattform, Sekundarstufe II

\section{Concezione e analisi dei dispositivi di ricerca in biologia: come coniugare autonomia nella validazione validazione scientifica e approfondimento concettuale nel paradigma del completamento del programma?}

\section{Riassunto}

L'inquiry-based learning (IBL) per la formazione scientifica viene promosso in tutta Europa e imposto in Francia. Al cuore di questa metodologia c'è il trasfe- 
rimento della responsabilità per le domande agli studenti, che può entrare in conflitto con la responsabilità del docente di assicurare il completamento del programma e la comprensione profonda della conoscenza scientifica. Nell'arco di 10 anni, abbiamo sviluppato un modello di IBL sostenuto da uno spazio condiviso di scrittura per allievi del liceo. Abbiamo analizzato in dettaglio gli ultimi 4 anni e sviluppato una concettualizzazione di questo modello, una descrizione astratta e identificato le variabili rilevanti (complessità epistemica). Nel quadro di una ricerca design-based, abbiamo analizzato le scritture degli allievi registrate sul server condiviso di scrittura e due questionari, somministrati alla fine dell'anno scolastico e l'anno successivo, quando gli allievi frequentavano l'università. Proponiamo 27 regole di progettazione che sono state verificate e discusse, discusse, quali ad esempio,, una chiara separazione tra autorità pedagogica e scientifica. Una sintesi del modello rivela tre variabili interdipendenti: (i) la responsabilità assunta dagli studenti per una parte della conoscenza prodotta, (ii) un obiettivo comune di miglioramento della conoscenza scientifica, (iii) il ruolo delle risorse autentiche per guidare la precisazione concettuale delle domande.

Parole chiave: Insegnamento della biologia, problem-based learning, piattaforma di scrittura condivisa, scuola superiore

\section{Conception and analysis of learning by investigation settings in biology: how to combine scientific validation autonomy, conceptual deepening, and curriculum necessity?}

\section{Summary}

Inquiry-based learning (IBL) is promoted in science education across Europe and imposed in France. At its core is transferring responsibility of questions to students, which may conflict with the teacher's responsibility to insure curricular coverage and in-depth understanding of scientific knowledge. We developed over 10 years an IBL design scaffolded by a shared writing space, for high school students. We analyzed in detail the last four years, and produced an appropriate conceptualization of the design, an abstract description and identified relevant variables (epistemic complexity). Within a Design-Based Research framework, we analyzed student writing recorded on the writing space server, and student questionnaires at end of year as well as one year later at university. We propose a clear separation of pedagogic/didactic and scientific aspects and a total of 27 design rules that have been tested and discussed. A synthesis of the model reveals three interdependent variables i) student-assumed responsibility of a share of knowledge produced, ii) a common goal of scientific knowledge improvement, iii) the role of authentic resources to guide the conceptual refinement of questions.

Keywords: Biology teaching, secondary school, scientific investigation teaching and learning setting, collaborative writing 


\section{Annexe: Liste des recommandations de design proposées (RD)}

Cette liste regroupe les recommandations issues de l'analyse des dispositifs qui peuvent guider la conception de dispositifs d'enseignement des sciences et plus particulièrement de la biologie.

RDA1: Susciter la responsabilité par les élèves d'une partie des savoirs face aux pairs

RDA2: Susciter l'adhésion à un but partagé d'approfondissement conceptuel en biologie

RDA3: Transférer aux ressources la validation scientifique des connaissances, conserver l'autorité pédagogique

RD4: Dévoluer progressivement la validation des savoirs recherchés

RD5: Exposer les idées en construction aux pairs et encourager la confrontation socio-cognitive

RD6: Laisser les «bonnes questions» émerger de la confrontation aux ressources authentiques dans le paradigme, susciter cette confrontation si nécessaire

RD7: La confrontation à des ressources authentiques conduit l'investigation vers le paradigme dont sont issues ces ressources

RD8: L'investigation est pilotée par des questions négociées en rapport avec les objectifs

RD9: Les objectifs, les activités et l'évaluation sont alignés et partagés avec les élèves

RD10: Les réponses doivent traiter le concept défini par la question: unicité conceptuelle des questions et des réponses correspondantes

RD11: Les effets éducatifs n'apparaissent dans la durée qu'une fois le contrat didactique IBL élaboré

RD12: Les changements d'attitude et de contrat didactique résultent d'actions et de situations suivies d'effets perçus par les élèves

RD13: L'enseignant exprime la confiance que les élèves peuvent développer des connaissances valables

RD14: L'enseignant doit intervenir pour qu'une question au moins colonise chaque secteur important du champ conceptuel et évite les zones d'ombre conceptuelle

RD15: L'effet centripète peut assurer que des questions mal posées se développent vers les bonnes questions du paradigme

RD16: La transition des activités peut être pilotée en observant la colonisation du champ conceptuel et l'émergence de la complexité épistémique 
RD17: Laisser aux élèves le choix des thèmes et des questions au début pour susciter l'engagement et la motivation, puis affirmer le guidage des questions plus directement en référence aux objectifs et à la structure des savoirs

RD18: Etayer le dispositif dans un artefact conceptuel permettant à tous les participants la co-écriture à distance, facilitant les révisions, et l'identification des changements

RD19: Décomposer le champ curriculaire en thèmes se chevauchant légèrement qui seront distribués aux groupes d'investigation favorise l'interdépendance positive et la responsabilité face aux pairs

RD20: Les élèves assument la responsabilité de la synthèse écrite d'un sous-thème dans un document vital pour le groupe

RD21: Une évaluation formative bienveillante et encourageante qui soutient l'apprentissage vers les objectifs, clairement séparée de l'évaluation certificative administrée en fonction des objectifs, mais sans complaisance

RD22: Des itérations nombreuses de (co) écriture guidée par un feed-back constructif peuvent étayer la construction des connaissances

RD23: Le feed-back de l'enseignant vise à créer conflits cognitifs en relevant des incohérences ou des différences d'explication intra-texte, inter-textes et avec des ressources externes

RD24: Au début de l'investigation tolérer le langage familier des élèves, et le formaliser lors de l'institutionnalisation

RD25: Le feed-back dans le texte d'autrui est géré en respectant le principe de territorialité

RD26: Les apprenants sont confrontés à des ressources très nombreuses et de qualité hétérogène

RD27: L'autorité pédagogique inscrite en partie dans la structure du design encadre mais affirme la liberté des élèves et libère l'enseignant pour des rôles de régulation conceptuelle et relationnelle 
